\title{
A Time-Space Network Optimization Model of Inter-city Train Operation for Energy Saving based on Circulation Mileages
}

\author{
Hui HU ${ }^{1, a}$ Chun-hua YE ${ }^{2, b}$ \\ ${ }^{1}$ School of Economics and Management, East China Jiaotong University, P.R.China \\ ${ }^{2}$ School of Railway Transportation, East China Jiaotong University, P.R.China \\ ahh24895@163.com \\ b1042067119@qq.com
}

Keywords: inter-city train; time-space network; circulation miles; optimization model.

Abstract. From point view of operation and management, a time-space network optimization model of inter-city train operation for energy saving on a high speed rail line is proposed on the basis of train time table predetermined using rail stock circulation mileages. The model's objectives are to minimize circulation of rail stock and total energy consumption, and decision variables are number of train units in stations, while constraints include node flow conservation, passenger demand and capacity limitation. Finally, a referenced simulation case is provided and solved for comparison and an optimization analysis is carried on to illustrate the model's feasibility and effectiveness.

\section{Introduction}

Generally speaking, energy consumption is considered as one of core indexes both for the transportation industry and society. Although railway transportation system is more energy efficient than most other transportation systems, the issue of raising energy efficiency for railways is focused to further reduce their contribution to climate change [1]. Energy efficiency for railways is mainly concerned with saving energy consumption and this will reduce energy costs and emissions negative impacts.

Saving energy consumption can be categorized primarily into technology approach and management approach [2-5]. Technology approach includes new energy introduction, locomotive and railway stock reengineering, train hauling manipulation strategy, train energy consumption estimation et al. Management approach involves energy saving management evaluation and optimization et al.

In this paper, we employ the management approach to develop energy efficient train operation control strategy based on time-space network model for inter-city passenger rail transportation on a high speed rail line. First, on the basis of some relevant papers and assumptions[6,7], a time-space network formulation of train operation strategy is constructed using train timetable and an energy consumption optimization model based on rail stock circulation miles is put forward. Then, a simulation case is referenced and validated in different scenarios so that energy saving efficiency can be compared. Finally, conclusions are summarized and possible further researches are given.

\section{Energy Efficient Train Operation Optimization Model based on Time-space Network}

\section{Time-space network model introduction}

From a given timetable such as table 1, a train operation time-space network model can be built as follows. The node is defined as $(\mathrm{S}, \mathrm{T})$ and the edge is composed of the corresponding nodes' relationship. For the node, $\mathrm{S}$ is station while $\mathrm{T}$ is the train arrival and departure time which can be got from train timetable. For the edge, there are 3 kinds of edges in the network model. First is the relation between $(x, t)$ and $\left(x^{\prime}, t^{\prime}\right)$ for a train, this means a train leaves station $x$ at time $t$ and arrives station $x^{\prime}$ at time $t^{\prime}$. Second is the relation between $(x, t)$ and $\left(x, t^{\prime}\right)$ for all trains in successive times $t$ and $t^{\prime}$ in the same station. Third is the relation between $(x, t)$ and $\left(x, t^{\prime}\right)$ for trains where $t$ is referred to the latest departure or arrival time in a station $\mathrm{x}$ and $\mathrm{t}$ ' is referred to the earliest departure of arrival time in a station x. The time-space network model can be depicted as Fig.1. 
Table.1 Train timetable on a line

\begin{tabular}{|c|c|c|c|c|}
\hline Time & $\mathbf{T r} \_1$ & Tr_2 & ...... & Tr_m \\
\hline Station S1 & T1_1d & T2_1d & $\ldots \ldots$ & Tm_1d \\
\hline Station S2 & $\begin{array}{l}\text { T1_2a } \\
\text { T1_2d }\end{array}$ & $\begin{array}{l}\text { T2_1a } \\
\text { T2_2d }\end{array}$ & $\ldots \ldots$ & $\begin{array}{l}\text { Tm_1a } \\
\text { Tm_2d }\end{array}$ \\
\hline$\ldots \ldots$ & $\ldots \ldots$ & $\ldots \ldots$ & $\ldots \ldots$ & $\ldots \ldots$ \\
\hline Station Sn & T1_na & T2_na & $\ldots \ldots$ & Tm_na \\
\hline
\end{tabular}

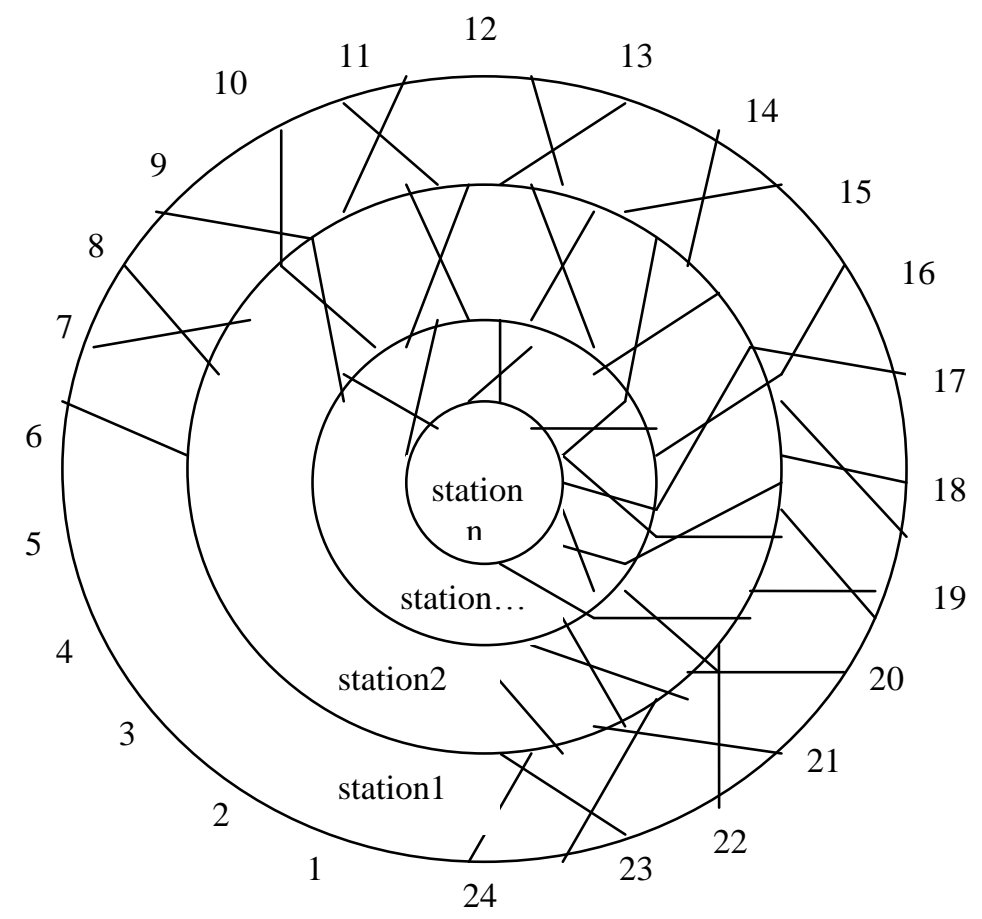

Fig.1 Train operation time-space network diagram

\section{Assumptions}

In order to formulate and solve the model, several assumptions are made as follows:

(1) The length of train can be extended or shortened in the station on a railway line, while constricted to same kinds of rail stock unit.

(2) Rail stock's energy consumption is proportional to its running distance, independent of passengers in it.

\section{Sets}

$S$ station set; $L$ segment set; $I$ train number set; $J$ train unit set; $T$ train time table set; $C$ passenger category set; $s$ 1 origin and destination station set of train; $V(s, t)$ vertices set; $G(s, t, s, t)$ arcs set; $G O(s, t, s, t)$ overnight arcs set.

\section{Parameters}

$T_{-} S_{s, t_{-} a}^{i}$ arrival time of train $i ; T_{-} S_{s, t_{-} d}^{i}$ departure time of train $i$; $O D_{s 1, s 2}^{i, c}$ OD passenger $c$ flow from station $S 1$ to station $S 2$ for train $i$; Dist ${ }_{s 1, s 2}$ distance between $S 1$ and $S 2$; Carriage ${ }_{j}$ carriage numbers for train unit $j$; Cap_Train ${ }_{j}^{c}$ seat category $c$ capacity of train unit $j$; Cap_Station maximum

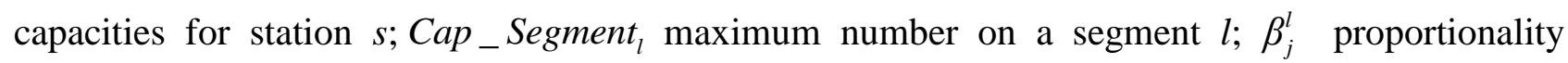
coefficient of train unit $j$ on segment $l$. 


\section{Variables}

$x_{g}^{j}$ number of arc $g$ in $G$ for train unit $j$; Energy $y_{l}^{i, j}$ energy consumption of train unit $j$ in train $i$ on segment $l$.

\section{Constraints}

$$
\begin{aligned}
& \sum_{g \in \delta^{+}(v)} x_{g}^{j}=\sum_{g \in \delta^{-}(v)} x_{g}^{j}, \forall v, j \\
& \sum_{i} O D_{g}^{i, c} \leq x_{g}^{j} * C a p_{-} \operatorname{Train}_{j}^{c}, \forall c, j \\
& x_{g}^{j} * \text { Carrigae }_{j} \leq \max \left(\text { Cap_Station }_{s}, \text { Cap_Segment }{ }_{l}\right) \\
& \text { TotalDis } \tan c e_{l}^{i, j}=x_{i s}^{j} * \text { Dis } \mathrm{t}(\mathrm{s} 1, \mathrm{~s} 2)
\end{aligned}
$$

Constraint (1) assures that train unit flow conservation. Constraint (2) and (3) satisfies the demand and capacity requirements. Equation(4) is used to compute the distance between $(\mathrm{s} 1, \mathrm{~s} 2)$ for rail stock unit $\mathrm{j}$ in train $\mathrm{i}$.

\section{Objective functions}

$$
\begin{aligned}
& \text { Minimize } \sum_{j} \sum_{g \in G 0} x_{g}^{j} \\
& \text { Minimize } \sum_{j} \sum_{i} \sum_{L} \text { TotalDis } \tan c e_{l}^{i, j} * \beta_{j}
\end{aligned}
$$

Objective function (5) is to minimize the total number of train units deployed for the overnight arcs, while function (6) is to minimize the total energy consumption for the all train units.

\section{Model Solution Algorithm}

Obviously, this model is an integer programming model and it's inherently a minimum-cost circulation problem whether the model's objective is the total number of train units or total energy consumption. So, we can apply standard min-cost circulation algorithms to solve it ${ }^{[8]}$ and GAMS software is used to develop the model and the solver is CPlex in this paper.

\section{Simulation Case Study}

\section{Case Description}

To confirm this model's validity, a referenced simulation case ${ }^{[7]}$ is studied and compared. That's to say, there're 36 trains on an inter-city high speed line. All the trains' timetable is given, so is for the passenger flow between the two stations on the line. In order to compute the rail stock unit's circulation miles, the distances between the neighbor stations are assumed to be $400 \mathrm{~km}$ (S1-S2), $200 \mathrm{~km}$ (S2-S3), 300km (S3-S4) along the line. Also, only on type of rail stock unit is available and $\beta_{j}=70 \mathrm{~kW} \cdot \mathrm{h} / \mathrm{km}$.

\section{Optimization Results Analysis}

For the purpose of illustrating the energy saving efficiency in the model, two sub-models are solved separately. Their objectives are to minimize total train units in overnight arcs, total train energy consumption respectively. The optimization models' results are depicted in Table 2.

.Table 2 Optimization results comparison

\begin{tabular}{c|c|c|c|c}
\hline $\begin{array}{c}\text { Minimizing } \\
\text { Objective Function }\end{array}$ & $\begin{array}{c}\text { Total Train } \\
\text { Unit } \\
\text { Circulations }\end{array}$ & $\begin{array}{c}\text { Total } \\
\text { Circulation } \\
\text { Miles (km) }\end{array}$ & $\begin{array}{c}\text { Energy } \\
\text { Consumption } \\
(\mathbf{k W . h})\end{array}$ & $\begin{array}{c}\text { Energy } \\
\text { Saving } \\
\text { Efficiency }\end{array}$ \\
\hline Overnight train units & 22 & 83800 & 5866000 & - \\
Circulation mileages & 25 & 74400 & 5208000 & $11.22 \%$ \\
\hline
\end{tabular}


As seen in Table 2, raising train units can save energy consumption and adjusting train unit's circle number in a reasonable manner help improve energy saving for their contribution to shortening circulation mileages, but increasing energy efficiency seems limited to some extent.

\section{Conclusion}

Along with sustainable development put forward, efficient energy use in rail transportation is heightened. Using circulation miles of rail stock unit, an optimization model of train operation for energy saving on the basis of time-space network formulation is employed and developed in this paper. Not only validated in the simulation case for the model feasibility and effectiveness, energy saving efficiency is illustrated by comparing with different scenarios. As for further research, more accurate energy consumption methods can be taken into consideration on one hand, this model is able to be extended to network comprised of lines that share the same stock of railway material on the other hand.

\section{Acknowledgements}

This work was financially supported by Soft Science Research Base on new industrialization and urbanization of Jiangxi Province and $\mathrm{PhD}$ Start-up Fund of East China Jiaotong University $(24341055,26441001)$.

\section{References}

[1] The Case for Business Investment in High-speed and Intercity Passenger Rail: American Public Transportation Association. February, 2011.

[2] HU Hui. A Research on Energy Saving in China's Railway Transportation System. Journal of East China Jiaotong University, 2011, 28(6): 73-79.

[3] Howlett P G. Optimal Strategies for the Control of a Train. Automatica, 1996,32(4):519-532.

[4] JIN Weidong, WANG Zili, LI Congwei et al. Study on Optimization Method of Train Operation for Saving Energy Journal of the China Railway Society, 1997, 19(6): 58-62.

[5] Li Zhiyong, Wen Rui, Wei Rongyong. Study on Locomotive Traction Energy Consumption Calculation Based on RBF Neural Network[J]. Journal of the China Railway Society, 2011, 33(9):27-30.

[6] XUE Yanbing, WANG Lie, CHEN Zhirong. EMU Energy Consumption Calculation and Analysis of Guangzhou-Shenzhen PDL. Railway Transport and Economy, 2011, 33(7):39-42.

[7] A. Schrijver. Minimum Circulation of Railway Stock, CWI Quarterly, 1993,(6): 205-217. 